\title{
Predictive control of Si content in blast furnace smelting based on improved SA-BP
}

\begin{abstract}
Blast furnace smelting process is a highly complex nonlinear dynamic process, its purpose is to refining the quality of liquid iron. From the point of view of chemical reaction kinetics, the main chemical reactions in the blast furnace are as many as 108 kinds, and the high complexity is obvious. From the point of view of hydrodynamics, there are three-phase mixed compressible viscous fluids in the blast furnace smelting process. The hydrodynamic equation is nonlinear, high-dimensional and high-coupling. In addition, the blast furnace smelting process has the characteristics of time-varying, high-dimensional, distributed parameters and other characteristics of the complex conditions and the failure of the operation under the conditions of the test, making the blast furnace smelting process automation and furnace temperature precision control to become metallurgical workers face the problem. In this study, from the data point of view, to explore the furnace temperature can be characterized by [Si] content prediction. Based on the data of 1000 furnace blast furnace, an accurate and reasonable prediction model of Si content in blast furnace is established. First of all, through the calculation of correlation coefficients of various factors and the time trend diagram, the general lag time between each parameter and Si content in the process of blast furnace production is obtained. Through correlation and analysis of the correlation between lag time, before and after the two largest furnace, with improved simulated annealing algorithm to determine the optimal initial weights, the content of Si, the N furnace of $S$ content, the quantity of coal and air PML FL as input, the contents of Si in $n+1$ furnace as output to establish dynamic prediction model of improved SA-BP neural network based. Finally, the data used to detect the model is brought into the model to be tested, the error is analyzed, and the error local map is used to express the error visually. The model is tested.
\end{abstract}

Keywords: Blast furnace; time lag analysis; BP neural network; improved simulated annealing algorithm.

\footnotetext{
Messrs. Wenhan Liu, Liye Shi, School of Mathematics and Physics, Handan College, Handan 056005 and Hongli Wang, Department of Mathematics and Information Sciences, Tangshan Normal University, Tangshan 063000, Hebei, China.
}

\subsection{Introduction}

$\mathrm{P}$ reparedness ensures success, unpreparedness spells failure. 'The main role of the forecast is to estimate and speculate on what is happening or not $[1,2]$. Accurate prediction can be regarded as the scientific basis for decision making. Prediction, served as an important tool, can completely reflect human subjective initiative, and the prediction has played an essential role in human daily production.

'Made in China 2025' is a grand strategy for China's manufacturing industry to upgrade. Its core technology is intelligent manufacturing, and iron and steel, metallurgy, petrochemical and other industries are representative of the pillar industries for the national economy. Take smelting of high quality molten iron in blast furnace for example. Intelligent control over the process of the blast furnace production is an important research subject on modern iron and steel metallurgy. For complex metallurgical processes, accurate prediction is an important prerequisite for good performance. Si content during the blast furnace smelting is an important index to reflect the complex physical and chemical state in the blast furnace. It is affected by many factors such as raw materials and operation ${ }^{[3,4]}$.

For a long time, many workers have put forward a variety of prediction models of Si content in blast furnace to judge the content of $\mathrm{Si}$, The purpose is to better control the furnace temperature, making the blast furnace smelting to be smooth and stable. These prediction models can be roughly divided into three categories: Semi empirical model combined with heat balance [5-7]; Empirical model combined with statistics [8]; Artificial Intelligence (Abbreviated as AI) model based on Artificial Neural Network [9] (Abbreviated as ANN). Because the environment of blast furnace smelting is becoming more and more complex, semi empirical and empirical model has been difficult to ensure the accuracy of prediction and effective control over the production process.

With the neural network model developing, more and more workers begin to establish the prediction model of Si content in blast furnace by using the powerful mapping ability of neural network [9]. At present, the neural network prediction model at home and abroad is mainly confined to the error 
propagation network which distinguishes the working state and learning state (Abbreviated as BP Network). It is a new attempt to explore the initial weights and various parameters about the network, which will provide a new idea for the prediction method.

\subsection{Time delay analysis of blast furnace smelting}

\subsection{Control MEChANiSM OF BLAST FURNACE SMELTing}

In the process of smelting blast furnace, due to the different production conditions, the control range of allowable furnace temperature is different for different blast furnaces, but generally the range of control is wide, which reflects the flexibility of the operation control of the blast furnace. However, such a wide range of control is only the feasible operation range of blast furnace operation, and cannot be used as the optimal control target of furnace temperature predictive control. The so-called furnace temperature control optimization refers to the temperature of the midline control, that is, in the best control range of furnace temperature finetuning. The optimal control range of furnace temperature, generally for different blast furnace or different operation objectives, the solution results will be different. The multi target in the multi objective programming of blast furnace smelting process, usually refers to: improved utilization coefficient, reduced coke ratio, the sulfur content index of hot metal meets the requirement of steelmaking and stable operation of furnace temperature.

After a long-term research of the scientific research workers, combined with the practice of blast furnace production and the principle of metallurgical reaction engineering, the qualitative requirement of blast furnace smelting process is transformed into quantitative mathematical model, which reflects the multi-objective mathematical model of operation optimization control of blast furnace.

Solving multivariate variation ally process functions: $F_{1}$, $F_{2}, F_{3}, F_{4}$, under the condition of satisfying (3), (4) and (4), maximizing the formula (1), and minimizing the formula (2): (1)

$$
\begin{aligned}
& U(t)=\int_{t_{0}}^{t} F_{1}\left(\boldsymbol{Y}, \frac{\partial \boldsymbol{Y}}{\partial \tau}, \boldsymbol{Z}, \frac{\partial \boldsymbol{Z}}{\partial \tau}, \boldsymbol{X}, \frac{\partial \boldsymbol{X}}{\partial \tau}, \boldsymbol{u}, \frac{\partial \boldsymbol{u}}{\partial \tau}\right) d \tau \rightarrow \max \\
& K(t)=\int_{t_{0}}^{t} F_{2}\left(\boldsymbol{Y}, \frac{\partial \boldsymbol{Y}}{\partial \tau}, \boldsymbol{Z}, \frac{\partial \boldsymbol{Z}}{\partial \tau}, \boldsymbol{X}, \frac{\partial \boldsymbol{X}}{\partial \tau}, \boldsymbol{u}, \frac{\partial \boldsymbol{u}}{\partial \tau}\right) d \tau \rightarrow \min \\
& {[\mathbf{S i}](t)=\int_{t_{0}}^{t} F_{3}\left(\boldsymbol{Y}, \frac{\partial \boldsymbol{Y}}{\partial \tau}, \boldsymbol{Z}, \frac{\partial \boldsymbol{Z}}{\partial \tau}, \boldsymbol{X}, \frac{\partial \boldsymbol{X}}{\partial \tau}, \boldsymbol{u}, \frac{\partial \boldsymbol{u}}{\partial \tau}\right) d \tau \in\left([\mathbf{S i}]_{0}-A,[\mathbf{S i}]_{0}+A\right)} \\
& {[\mathbf{S}](t)=\int_{t_{0}}^{t} F_{4}\left(\boldsymbol{Y}, \frac{\partial \boldsymbol{Y}}{\partial \tau}, \boldsymbol{Z}, \frac{\partial \boldsymbol{Z}}{\partial \tau}, \boldsymbol{X}, \frac{\partial \boldsymbol{X}}{\partial \tau}, \boldsymbol{u}, \frac{\partial \boldsymbol{u}}{\partial \tau}\right) d \tau \leq[\mathbf{S}]_{0}}
\end{aligned}
$$

In the above model: $Y$ represents the raw material variable $\boldsymbol{Y}(t)=\left(y_{1}(t), y_{2}(t), \ldots, y_{m}(t)\right)$ of blast furnace in real time; $\boldsymbol{X}$ represents the detectable state variable in the smelting process, this variable is monitored in time, and is mainly used to infer the smelting state of blast furnace, $\boldsymbol{X}(t)=\left(x_{1}(t), x_{2}(t), \ldots\right.$, $\left.x_{n}(t)\right) ; \boldsymbol{Z}$ represents the equipment variables, that is, the state parameters of the blast furnace body and auxiliary equipment, most of these variables are relatively fixed, but there are also partial variations of the parameters, $\boldsymbol{Z}(t)=\left(z_{1}(t), z_{2}(t), \ldots, z_{l}(t)\right)$; $u$ is the control variable, which is a controllable variable. It is mainly used to adjust these variables, change the smelting status in the furnace, achieve the steady state variables, and achieve the final quality control of molten iron, . $\boldsymbol{u}(t)=\left(u_{1}(t)\right.$, $\left.u_{2}(t), \ldots, u_{n}(t)\right)$,

Formula (1) means maximization of the efficiency of blast furnace, that is, maximizing the coefficient of utilization $U(t)$; formula (2) said that the minimum of energy consumption of per tonne iron, that is, coke ratio of $K(t)$ is minimized; formula (3) represents the best control requirement of the key parameter $[\mathrm{Si}]$ in the process. $\left([\mathrm{Si}]_{0}-A,[\mathrm{Si}]_{0}+A\right)$ represents the optimum control range of furnace temperature, and $[\mathrm{Si}]_{0}$ is its "control center line"; The formula (4) indicates that the content of sulphur in molten iron must meet the quality requirement of steelmaking process. The formula (3) is the necessary condition for stabilization, balance and direct running of blast furnace smelting process. The constraint function $R(t)$ represents the material balance (Alkalinity optimization), and $H(t)$ represents heat balance (load optimization).

To achieve the blast furnace temperature prediction control, the primary task is to establish an accurate and available blast furnace temperature prediction model, the operator controls the blast furnace timely and moderately, which is based on the prediction of the mathematical model. Only through the mathematical model to grasp this trend, intelligent control based on furnace temperature stability development can be realized. The temperature of stable development, which can reduce the unnecessary heat loss, reduce the coke ratio, improve the utilization factor and reduce the overall fuel ratio. Naturally, the control goal of "high quality, low consumption, high production and long life" can be achieved, "safe, stable, balanced, direct" operation of the blast furnace is also easy to implement. By understanding the complexity of process mechanism and complexity of smelting process in the first two sections, the target of furnace temperature control can be objectively determined, and the optimal control of furnace temperature based on process mechanism and mathematical model is realized.

In this study, we collected the detection value of silicon content in blast furnace molten iron under 1000 time series and the [S] content, coal consumption and blast volume corresponding to the time synchronization. Part of the data are given in Table 1:

Due to some production characteristics of the blast furnace itself, there is a certain time lag effect in the amount of pulverized coal injection PML, the amount of blast FL and the content of $\mathrm{Si}$ in molten iron. This effect should be 
TABle 1: Statistical list Of Silicon CONTENT IN HOt METAL OF BlaSt FURNACE UNDER TIME SERIES

\begin{tabular}{|c|c|c|c|c|}
\hline Time series & {$[\mathrm{Si}]$} & {$[\mathrm{S}]$} & PML & $\mathrm{FL}$ \\
\hline 1 & 0.48 & 0.02 & 12.14 & 1644.31 \\
\hline 2 & 0.5 & 0.01 & 11.62 & 1652.88 \\
\hline 3 & 0.42 & 0.01 & 10.32 & 1703.58 \\
\hline 4 & 0.41 & 0.012 & 12.05 & 1707.49 \\
\hline 5 & 0.48 & 0.01 & 12.92 & 1728.84 \\
\hline 6 & 0.6 & 0.015 & 12.01 & 1736.26 \\
\hline 7 & 0.42 & 0.023 & 14.43 & 1692.77 \\
\hline 8 & 0.4 & 0.019 & 13.85 & 1691.13 \\
\hline 9 & 0.47 & 0.016 & 14.48 & 1681.49 \\
\hline 10 & 0.4 & 0.01 & 12.58 & 1666.51 \\
\hline 11 & 0.7 & 0.012 & 12 & 1691.81 \\
\hline 12 & 0.52 & 0.02 & 13.81 & 1573.18 \\
\hline 13 & 0.7 & 0.013 & 11.59 & 1665.97 \\
\hline 14 & 0.48 & 0.019 & 8.02 & 1700.48 \\
\hline 15 & 0.54 & 0.023 & 10.21 & 1718.83 \\
\hline 16 & 0.49 & 0.015 & 10.59 & 1711.88 \\
\hline 17 & 0.58 & 0.011 & 11.63 & 1679.51 \\
\hline 18 & 0.6 & 0.013 & 11.92 & 1801.97 \\
\hline 19 & 0.53 & 0.016 & 13.86 & 1787.3 \\
\hline 20 & 0.62 & 0.01 & 17.18 & 1722.84 \\
\hline$\square$ & $\square$ & $\square$ & $\square$ & $\square$ \\
\hline 501 & 0.29 & 0.017 & 12.88 & 1815.98 \\
\hline 502 & 0.32 & 0.013 & 13.41 & 1820.6 \\
\hline 503 & 0.35 & 0.015 & 12.78 & 1802.39 \\
\hline 504 & 0.4 & 0.015 & 12.54 & 1813.16 \\
\hline 505 & 0.5 & 0.012 & 13.68 & 1820.47 \\
\hline$\square$ & $\square$ & $\square$ & $\square$ & $\square$ \\
\hline 990 & 0.5 & 0.015 & 13.63 & 1767.64 \\
\hline 991 & 0.6 & 0.015 & 12.86 & 1791.46 \\
\hline 992 & 0.68 & 0.014 & 13.16 & 1795.06 \\
\hline 993 & 0.52 & 0.015 & 14 & 1739.82 \\
\hline 994 & 0.54 & 0.011 & 13.68 & 1760.81 \\
\hline 995 & 0.35 & 0.018 & 13.64 & 1708.51 \\
\hline 996 & 0.3 & 0.016 & 13.72 & 1711.38 \\
\hline 997 & 0.51 & 0.01 & 11.34 & 1728.14 \\
\hline 998 & 0.44 & 0.012 & 12.2 & 1622.32 \\
\hline 999 & 0.46 & 0.026 & 11.04 & 1711.56 \\
\hline 1000 & 0.38 & 0.015 & 14.21 & 1679.44 \\
\hline
\end{tabular}

removed as much as possible before establishing the model, because $\mathrm{S}$ exists in molten iron with $\mathrm{Si}, \mathrm{S}$ has no time lag effect on $\mathrm{Si}[10]$.

\subsection{Pulverized coal injection Rate PML and Time lag ANALYSIS OF Si CONTENT}

To blow pulverized coal into blast furnace. Although it can increase the reducing gas and accelerate the reduction, on the other hand, decomposition of coal powder absorbs heat. Resulting in blast furnace temperature declining during the initial coal injection, until the fuel release heat after it works.
The time required to experience this process is the lag time[11], The formula is as follows:

$$
T=\frac{v_{1}}{v_{2}} \times \frac{1}{n}
$$

Note: $T$ is lag time $h$

$v_{1}$ is the total volume of the blast furnace outlet center line to the lower part of the furnace body, $\mathrm{m}^{3}$

$v_{2}$ The volume of each batch,

$n$ Charging times per hour

$$
T=\frac{v_{1}}{v_{2}} \times \frac{1}{n}
$$

By querying the information, for general, is $300 \mathrm{~m}^{3}, n$ is 11 , The average of $v_{2}$ is $13.16 \mathrm{~m}^{3}$, was calculated to be about 2 hours. Randomly select 10 consecutive sets of data, then draw the curve of the Si content and the amount of PML with time as is shown in Fig.1:

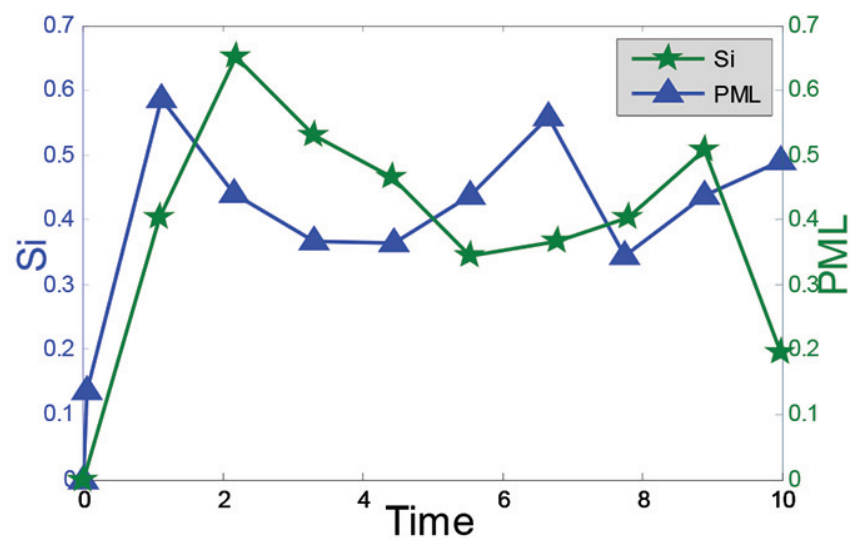

Fig.1: The time sequence diagram of Si and PML

It can also be seen from the figure that the lag time of the PML is about 2 hours, which is consistent with the calculated results.

\subsection{Analysis of time LAG of AIR VOlume FL AND Si CONTENT}

Air volume FL is very important for blast furnace ironmaking, which is beneficial to promote the rational distribution of air flow and improve the efficiency of coal injection [12]. At a certain point in time, temporary wind reduction can increase the furnace temperature namely $\mathrm{Si}$ content. Through the regulation of the air volume FL to adjust the furnace temperature, the fastest, the effect is also the most obvious [13]. But the blast furnace itself is a large hysteretic system, Therefore, there is delay time before air volume FL works out [14]. Randomly select 5 consecutive sets of data in the attachment, The correlation coefficients of the Si content in the current 1-5 group and the adjacent 6-10 group, 11-15 group and 16-20 group are shown in Table 2.

It can be seen from the table that the air volume FL and $\mathrm{Si}$ content, that is, negatively correlated with the furnace temperature, and the current air volume FL and the next 
TABle 2: The CORRELATION COEFFICIENT BETWEEN AIR VOLUME FL AND SI CONTENT

\begin{tabular}{lcccc}
\hline $\begin{array}{l}1-5 \text { Air } \\
\text { volume FL }\end{array}$ & $\begin{array}{c}1-5 \mathrm{Si} \\
\text { content }\end{array}$ & $\begin{array}{c}6-10 \mathrm{Si} \\
\text { content }\end{array}$ & $\begin{array}{c}11-15 \mathrm{Si} \\
\text { content }\end{array}$ & $\begin{array}{c}16-20 \\
\mathrm{Si} \text { 含量 }\end{array}$ \\
\hline 1644.31 & 0.43 & 0.60 & 0.70 & 0.57 \\
1652.88 & 0.50 & 0.42 & 0.52 & 0.65 \\
1703.58 & 0.46 & 0.40 & 0.70 & 0.61 \\
1707.49 & 0.48 & 0.47 & 0.48 & 0.53 \\
1728.84 & 0.45 & 0.40 & 0.54 & 0.62 \\
Coefficient & -0.025 & -0.636 & -0.313 & -0.123 \\
\hline
\end{tabular}

furnace temperature and negative correlation coefficient is the largest. As can be seen from the problem, the distance between each furnace is 2 hours, so the lag time for the air flow FL is about 2 hours.

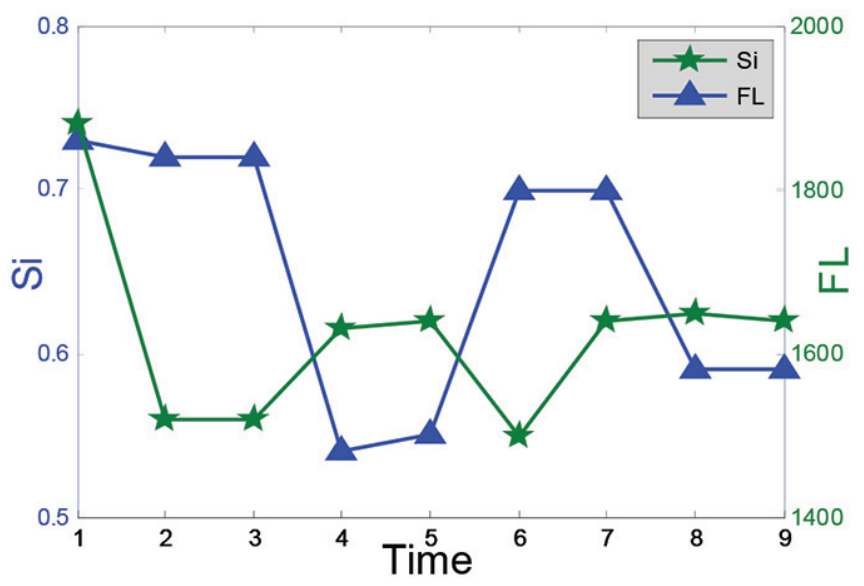

Fig.2 The time sequence diagram of $\mathrm{Si}$ and FL

Fig.2 is the sequence diagram of a random selection of continuous air flow FL and Si content, The lag time can also be seen from the figure, which is about 2 hours. On the one hand the wind can improve the furnace temperature, on the other hand the wind reduction will mean less production. Therefore the wind should be used to improve the furnace temperature only as a last resort.

\subsection{Basic theory of simulated annealing algorithm and its improvement}

\subsection{BASIC THEORY OF SIMULATED ANNEALING ALGORITHM}

The principle of simulated annealing algorithm is derived from the principle of solid annealing, and it is a general random search algorithm. The principle is to make solid heating temperature rise to sufficiently high, let it slowly cooling. When the solid is heated, with the increase of temperature inside the solid particles will shape into disorder and the internal energy can be increased. Slowly cool particles gradually towards the orderly. When each temperature has reached equilibrium state, finally the ground state at room temperature, the internal energy can be reduced to a minimum. There is a certain similarity between the optimization problem and the solid annealing process. Kirkpatrick $\mathrm{S}$ et al introduced the simulation which Metropolis et al. introduce the process of solids reaching the heat equilibrium at constant temperature into the optimization process [15]. That is, if:

$$
\Delta f=f(x(t+\Delta t))-f(x) \leq 0
$$

A new state is accepted, otherwise a new state is accepted according to probability $p(\Delta f)=e^{-\Delta f / T} \cdot T=T(t)$ is a parameter that decreases with time $t$ increasing, equivalent to the temperature during the annealing process. The use of similarity to solve the optimization problem and the physical system in the process of annealing, using Metropolis algorithm, appropriate controlling temperature drop process, the realization of simulated annealing and the random method that solves global optimization problem is called 'simulated annealing algorithm' [16].

$$
\Delta f=f(x(t+\Delta t))-f(x) \leq 0
$$

1. An initial solution is generated at random from the solution space $x_{0}$, and then calculate the corresponding objective function value. $f_{0}$, the initial solution has a great influence on the convergence speed and direction of the algorithm, so the initial solution can be regulated according to the specific situation in actual operation.

2. The new state generating function generates a new solution on the basis of the current solution, In order to simplify the calculation, the function which new solution can be generated by simple transformation is often used as the generating function, and differenence between the functions has a great influence on the speed of convergence and longitude. Therefore, we should choose carefully [18].

3. Calculate the objective function value of new solution $f_{1}$. If $f_{1}<f_{0}$, replace the current solution $x_{0}$ with a new solution $x_{1}$. Otherwise, the probability $e^{-\Delta t / T}$ decides whether to accept the new explanation or not.

4. Calculate the objective function value of the current solution $x_{0}$. If it is less than the tolerance, the algorithm stops and outputs the current solution. Otherwise repeats steps $1-3$, and cools at the same time until the conditional solution is generated [19].

\subsection{Algorithm IMPROVEMENT}

In this paper, we use binary coding to solve the solution space, and for the simulated annealing algorithm, this paper finds that the method of generating new solutions is too random and does not apply to the initial weight selection in this paper. Improve the process, the introduction of the selected probability of the concept, was selected probability calculation formula is as follows:

$$
Q_{i}=\frac{R_{(i)}}{\sum R_{(i)}}
$$

Where $Q_{i}$ represents the selected probability of the $i$-th 
bit, and $R_{i}$ represents the fitness after changing the $i$-th binary number.

And then randomly generate two numbers $r_{1}$ and $r_{2}$ between [0 1].

If $r<q_{1}$, the number $k_{1}$ on the first place is selected;

If $q_{m-1}<r<q_{m}$, the number $k_{m}$ is selected.

And then change the $m$-th digit $k_{m}$, generate a new solution $x$, for example, 101101 selected third, the new solution is 100101 .

The flow chart (Fig.3) is as follows:

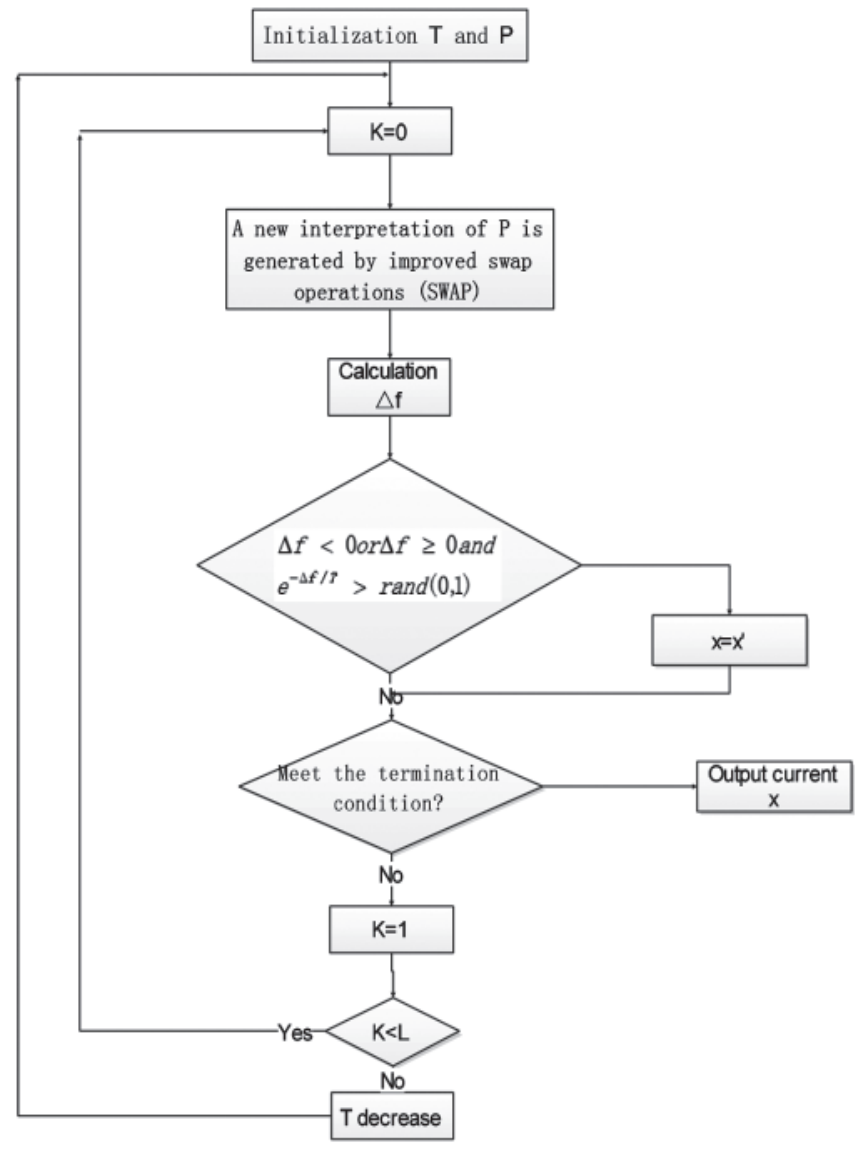

Fig.3 Improved simulated annealing algorithm flow

\subsection{Basic theory of BP neural network}

\subsection{BRIEF INTRODUCTION OF BP NEURAL NETWORK}

BP neural network is the abbreviation of error back propagation neural network, which consists of an input layer, one or more hidden layer and an output layer, each by a certain number of neurons. These neurons are as interrelated as human nerve cells [20]. Its structure is shown in Fig.4:

Let $w_{j i}$ be the connection weight between the neurons of the hidden layer and the $i$-th element of the input layer, and $v_{k j}$ is the connection weight between the $k$-th element of the output layer and the neurons of the hidden layer [21].

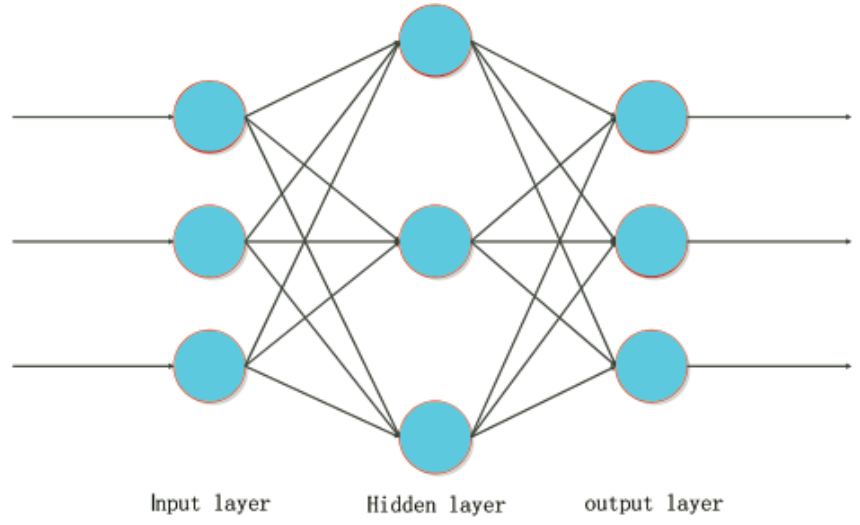

Fig.4 BP neural network model

\subsection{Parameter Setting of BP NeURAL Network}

There are no clear theoretical basis for the number of hidden nodes [22], generally based on empirical formula:

$l=\sqrt{m+n}+a$

or

$l=\sqrt{0.43 m n+0.12 n^{2}+2.54 m+0.77 n+0.35+0.51}$

Where $m$ and $n$ are the number of input nodes and the number of output nodes, respectively; and $\alpha$ is a constant between 1 and 10 .

Other parameters follow the general rules set to:

Learning rate: 0.05

Maximum number of training: 10000

Mean square error: $0.65^{*} 10^{\wedge}-8$

\subsection{Improved SA-BP algorithm}

5.1 Integration of Neural Network and Improved Simulated Annealing Algorithm

The simulated annealing algorithm can converge to the global optimal solution, and the improved simulated annealing algorithm has faster convergence speed and better robustness. Therefore, it is of some practical significance to combine the improved simulated annealing algorithm with BP neural network. Practical meaning: After combining, it not only can play a powerful mapping function of the neural network, but also can make the improved network learning ability is strengthened. The improved BP algorithm is optimized by the improved simulated annealing algorithm. The content includes three aspects: optimization of connection weight, optimization of network structure and optimization of learning rules.

\section{(1) Optimization of connection weights}

All the knowledge of the neural network system is basically included in the distribution of its connection weight, the general BP network weight is obtained through a certain search algorithm to modify the weight of the modified. By constantly adjusting the weights in the process of continuous 
training, the purpose is to get a set of smaller errors. This algorithm is a BP algorithm, but the shortcomings of the method is obvious, the most significant drawback is easy to fall into the local extremum and the convergence rate is slow, and many parameters set the need for a more rich experience. The improved simulated annealing algorithm can solve the problems caused by these shortcomings to a large extent, and find the optimal weights and thresholds.

\section{(2) Optimization of network structure}

A good network structure should be able to just solve the problem without the existence of redundant connections, the rationality of the network structure is directly related to the accuracy of the network. In the traditional BP network, the choice of structure is to rely on the user's experience to choose, the current structure of the choice of no scientific and effective way to solve, so the structure of the optimization is particularly important. In this paper, the use of a binary of a representative of an edge, if this bit is " 1 " on behalf of the existence of this side, " 0 " does not exist. And then convert the binary number into decimal, you can achieve the evolution of the network structure.

\section{(3) Optimization of learning rules}

The optimization of learning rules mainly includes two aspects: learning parameter optimization and learning rule optimization. There are many parameters in the learning process, and the optimization of these parameters can be optimized along with the network, or it can be optimized separately. The optimization of learning rules is aimed at the dynamic behaviour of neural network self-evolution. This research is at the initial stage and has some research prospects.

\subsection{The BASIC PRINCIPLES OF IMPROVED SA-BP ALGORITHM}

In contrast to the characteristics of the BP algorithm and the improved SA algorithm itself, the BP algorithm focuses on the modification of the weight value by the gradient descent method, which makes it easy to fall into some local minimum values: the improved simulated annealing algorithm. More focused on the global search capabilities, for local search becomes powerless. From the above two aspects, combined with BP and improved SA can achieve complementary advantages.

Using the improved SA to optimize the initial weight of the BP neural network, on the one hand, the selection range of the initial value can be made wider, and on the other hand, the convergence speed can be accelerated and the accuracy of the model can be improved. In this way, the training of the network is divided into two parts: first through the improved simulated annealing algorithm to optimize the initial weight of the network, and then through the BP algorithm to modify the weight of a small amount.

\subsection{IMPROVED SA-BP ALGORITHM STEPS}

The improved SA-BP algorithm steps are as follows:
1. Determine the input and output samples of the BP neural network.

2. To determine the network power factor of the new solution generation mode, select the annealing temperature, set the annealing parameters.

3. Select the network structure and learning rules. Randomly generate a set of weight values.

4. Calculate the error function of the neural network under different weights, and give the fitness function of the improved simulated annealing algorithm, the higher the fitness value is, the higher is the fitness value.

5. Use a new solution to produce the current weight value to adjust, resulting in a new solution.

6. Calculate the error of the new solution to determine whether the sample meets the requirements.

7. Repeat steps 4,5 , and 6 so that the weight distribution is continuously optimized until the accuracy is met.

\subsection{Prediction of Si content in blast furnace smelting}

\subsection{EXCEPTION OF ABNORMAL VALUE}

In dealing with data, often encounter individual deviation from the expected or a large number of statistical values of the situation, if these data directly with the normal data together to deal with, often resulting in a large deviation from the results, so this article first removes the abnormal data according to the formula:

$$
\begin{aligned}
& \text { Upper }=Q_{3}+2.2 *\left(Q_{3}-Q_{1}\right) \\
& \text { Lower }=Q_{1}-2.2 *\left(Q_{3}-Q_{1}\right)
\end{aligned}
$$

Use the spss to find the four influencing factors, respectively, the maximum critical value upper and the minimum threshold lower. Excluding data above and below lower, see Table 3 for data removal.

TABLE 3: THE REMOVED DATA TABLE

\begin{tabular}{ccc}
\hline & Serial number \\
\hline 48 & 507 & 822 \\
159 & 509 & 823 \\
210 & 512 & 824 \\
493 & 624 & 825 \\
\hline
\end{tabular}

\subsection{Selection of Training Samples}

According to the relevant information we can know [23] [24], the blast furnace Si content and the impact of the parameters of the furnace the most closely related, and by 5.2 time lag analysis can know the amount of coal PML, air flow FL hysteresis are 2 hours, you can see the impact of a furnace on the next furnace. To further verify the time lag, remove the first furnace after the 2-1000 furnace Si content and the former 1-999 furnace Si content, S content, the amount of coal injection PML, the amount of air FL correlation analysis.

When the significance is $<0.05$, the correlation is 
significant, the correlation is significant, and the correlation coefficient is large. Therefore, in this paper, four influencing factors of the $n$-th furnace are used as the input variables of BP neural network, and the Si content of the $n+1$ furnace is used as output, thus forming a sample, and 984 samples are used to form the sample set.

TABLE 4: CoRrelation COEFFICIENT OF Si WITH OtHER FACTORS

Si content S content Amount of Air volume coal injection

\begin{tabular}{lcccr}
\hline Correlation & & & & \\
coefficient & 0.631 & -0.231 & -0.543 & -623 \\
Significance & 0.01 & 0.02 & 0.03 & 0.01 \\
\hline
\end{tabular}

6.3 ESTABLISHMENT OF PREDICTION MODEL FOR Si CONTENT IN BLAST FURNACE SMELTING

At present, many mature theories and methods can be used to predict the content of $\mathrm{Si}$ in molten iron, such as partial least squares regression, regression model, etc.. But these theories and methods have certain limitations, and the artificial neural network model is currently popular particularly suitable for the non linear system, with inclusive, robust and so on [23][24], especially for the sample data is considerable problems with good feasibility, and the improved simulated annealing algorithm has the advantages of fast convergence and strong global solution ability [25], so this paper uses BP neural network and improved SA-BP model based on the content of $\mathrm{Si}$ was predicted and compared.

\subsubsection{Prediction model of Si content in blast furnace smelting based on $B P$ neural network}

Blast furnace smelting is a large hysteresis production process, its physical and chemical reactions, process parameters are numerous, it is difficult to find the Si content and the process of the exact relationship between the parameters. However, the BP network has a strong mapping ability, you can establish a complex relationship between multiple variables, in this paper, using MATLAB neural network toolbox to predict the content of $\mathrm{Si}$ and predictive test.

First, the training sample set into the first 200 groups into one, the remaining 784 components are divided into another, with a set of samples behind the sample training. Then the data is passed to MATLAB and the premnmx function is called to normalize the data. Based on experience, the formula calculates the number of hidden nodes to 10. Call the function newff to set up the network and train and save the trained network.

Training results are shown in Fig.5:

It can be seen from the simulation results that the prediction model of Si content in BP neural network has achieved some effect, but the accuracy of prediction is not enough.

6.3.2 Prediction model of blast furnace smelting Si content based on improved $S A-B P$

As the BP algorithm is easy to fall into the local minimum, slow convergence and other issues, this article has been improved, that is, first with improved simulated annealing initialization neural network weight, with the algorithm

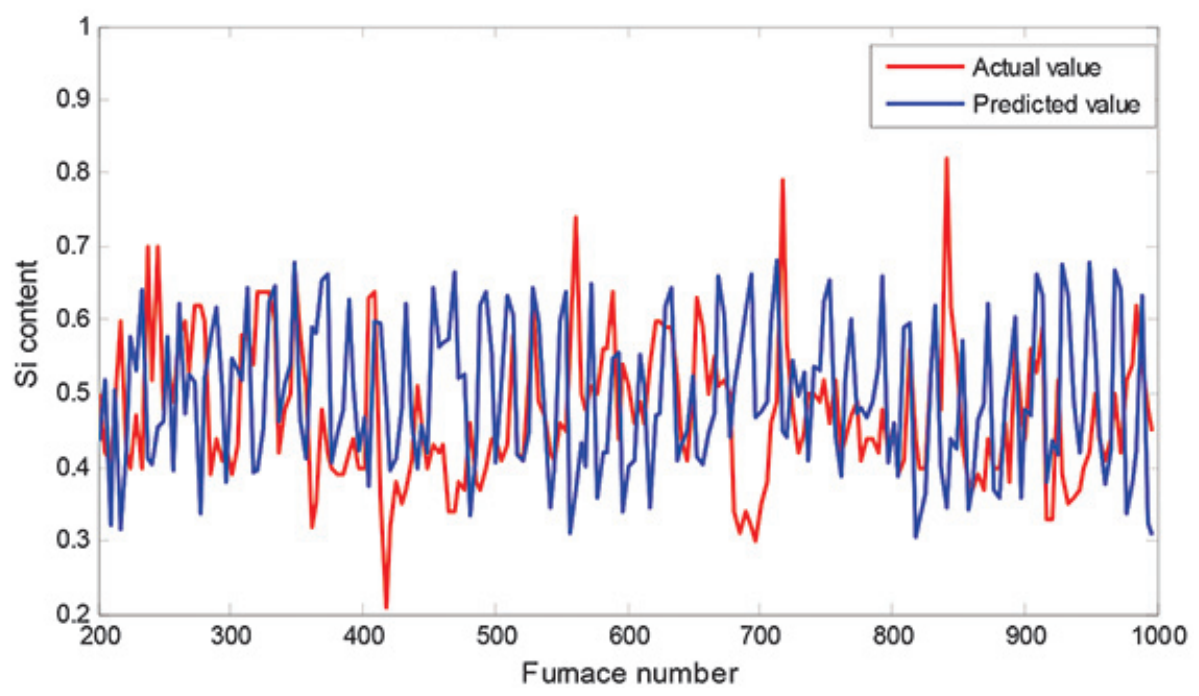

Fig.5: Predictive test

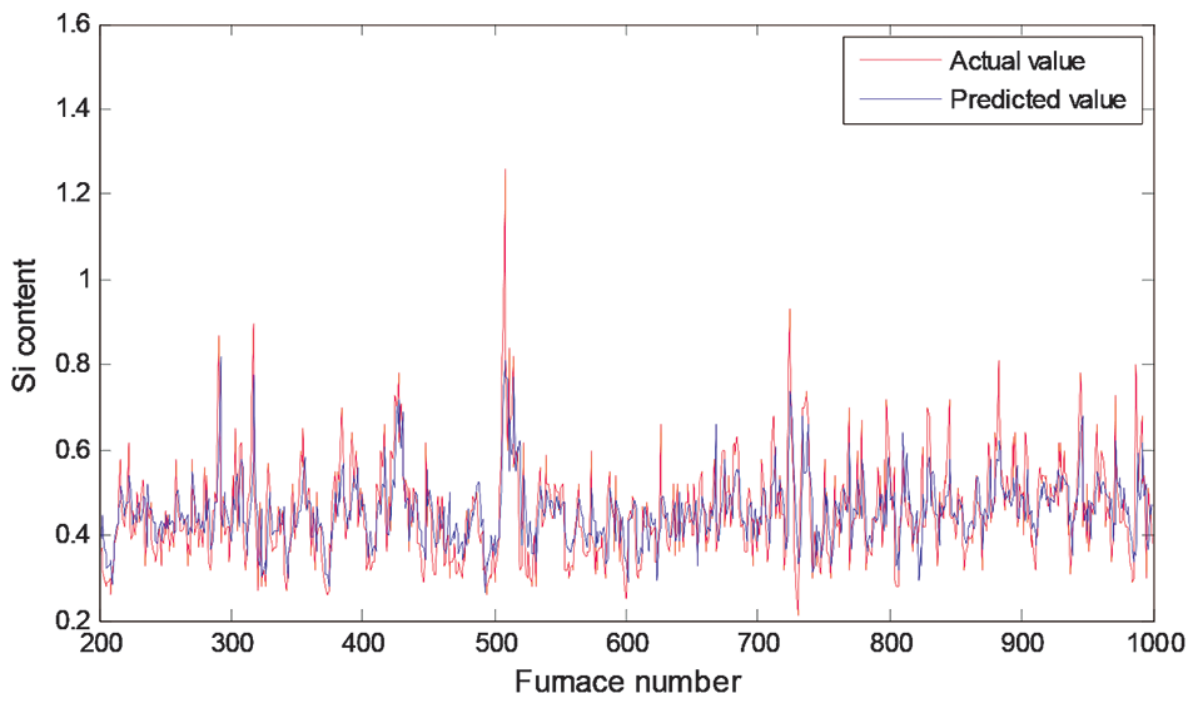

Fig.6: Predictive test 
learning and training. The initial temperature is 1000, and the code is programmed to optimize the initial weight until the error converges to the specified precision.

From the simulation results shown in Fig.6, with the improved simulated annealing algorithm, the prediction accuracy of Si content in blast furnace smelting has been greatly improved, and the convergence speed of the model is very fast.

\subsection{THE MODEL PREDICTS THE SUCCESS RATE}

For the two furnaces are correlated, the former 200 sets of data are selected as the verification samples, and the predicted mathematical model of $\mathrm{Si}$ is used to predict. For the data is too much, the image is not easy to express, so each prediction error will take absolute value, once every ten times to sum, and finally get 20 sets of error data, draw the error analysis shown in Figs.7 and 8:

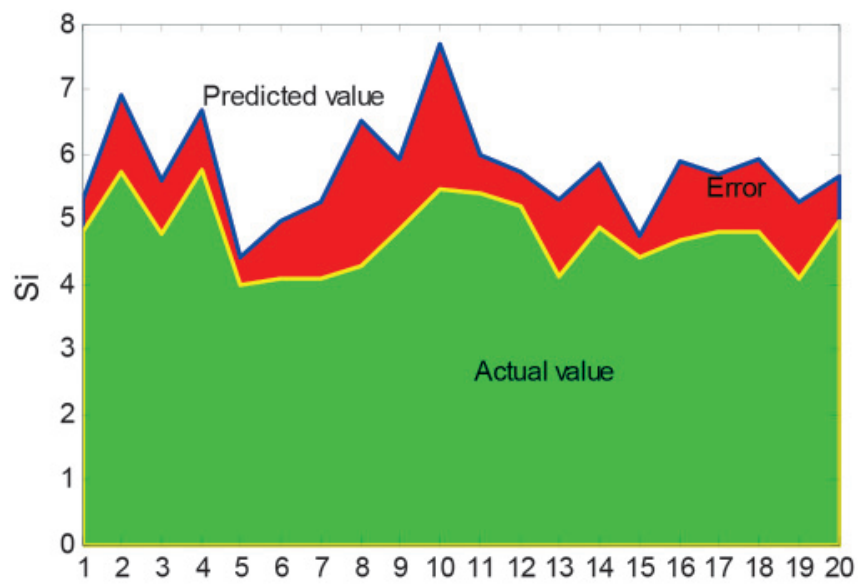

Fig.7: Common BP prediction model error analysis

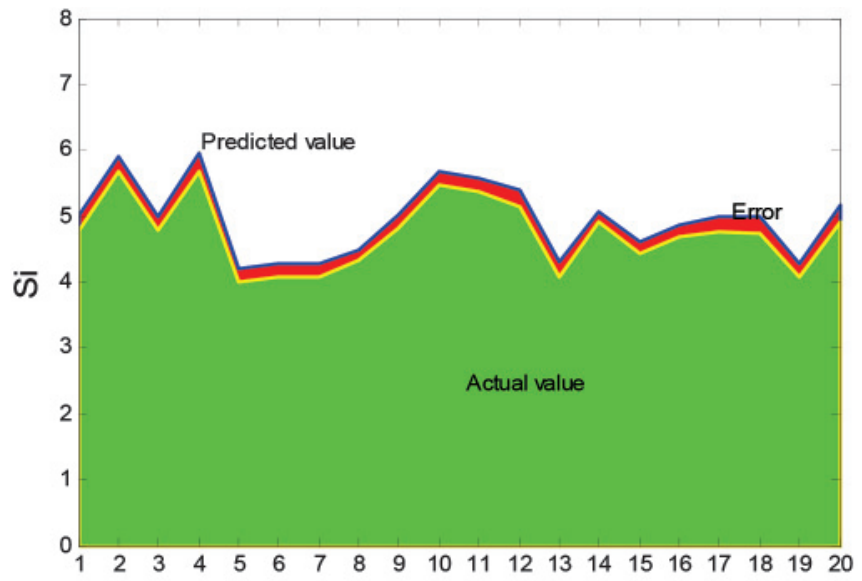

Fig.8: Based on improved SA-BP prediction model error analysis

In Figs. 7 and 8, red colour represents the error, green colour represents the exact value, it is easy to see, based on improved SA-BP prediction model compared to ordinary BP prediction model prediction effect is ideal.
The average absolute percentage error $E$ is used to represent the average of the absolute value of the ratio between the actual value and the predicted value and the actual value, the formula is:

$$
E=\frac{1}{n} \sum\left|\frac{y_{i}-y_{i i}}{y_{i}}\right|
$$

The average absolute error $E$ is $4.67 \%$ and the predicted success rate is $95.33 \%$, which indicates that the model is ideal.

If the predicted value between the two adjacent furnace and the actual value of the direction of the same, the record value is 1 , if the opposite, then the record value is 0 , according to 0 and 1 percentage of the total number of pie chart, the percentage of the value 1 is the predicted success rate of the furnace temperature rise and fall direction.

It can be seen from Figs. 9 and 10 that $86.5 \%$ of the furnace temperature rise and fall direction is consistent with the actual SA-BP prediction model, that is, the prediction success rate is $86.5 \%$, while the success rate of ordinary BP prediction model is only $71.5 \%$, the results show that the improved SA-BP prediction model is ideal.

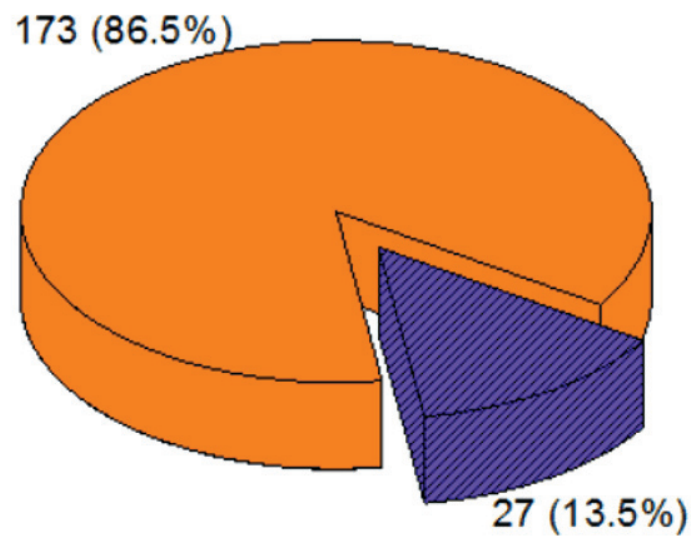

Fig.9: Common BP model furnace temperature prediction success rate

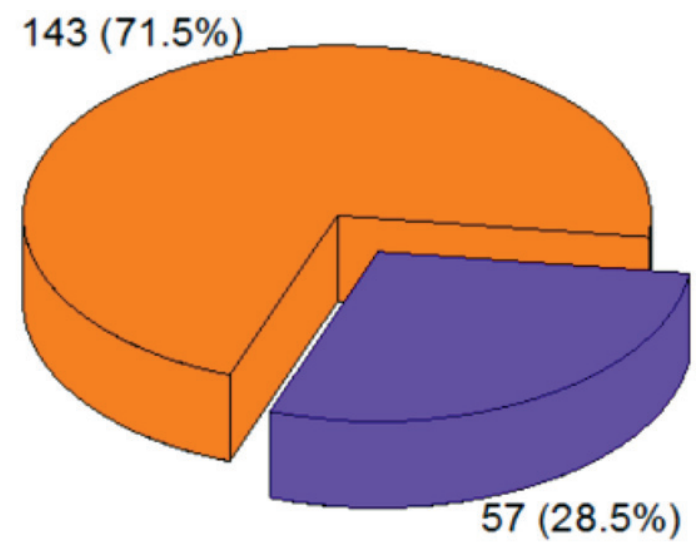

Fig.10: The furnace temperature prediction success rate based on the improved SA-BP model Note: where orange represents 1 per cent of the total 


\subsection{Conclusions}

According to the characteristics of blast furnace ironmaking, through time lag analysis, the correlation between variables is enhanced and the accuracy of model is improved. The BP neural network prediction model has strong nonlinear mapping ability and is suitable for solving the complicated internal mechanism [10][11]. It can predict the silicon content of molten iron in the blast furnace smelting process and the network has some generalization and generalization ability. It is very difficult for the nonlinear network to converge speed and global solution. Therefore, the simulated annealing algorithm is used to improve the BP neural network, and the more efficient annealing process is designed, combining with other mechanism algorithms, Improved simulated annealing algorithm to improve the budget speed, the results are more accurate than a single BP neural network, with a certain practical value.

\section{References}

[1] Yu Xiaofeng (2016): Machine learning theory in the railway freight forecasting application [D]. Beijing Jiaotong University.

[2] Yu Zhiheng (2016): . Based on the intelligent theory of traffic flow portfolio forecasting model [D]. Northeast Normal University.

[3] Zhao Zhe (2015): Blast furnace smelting process monitoring and trend forecast [D]. Inner Mongolia University of Science and Technology.

[4] Zeng Yanfei, Li Hushan. (2007): Design and implementation of predictive control model for [Si] content of blast furnace molten iron [J]. Microcomputer Information, (10): 64-66. [2017-08-02].

[5] Liu Juanli. (2017): Blast furnace heat balance analysis of ironsaving process energy-saving direction $[\mathrm{J}]$. Science and Technology Communication, 6 (11): 66-67. [2017-08-02].

[6] Zhang Guobo, Li Jian. (2017): Calculation and analysis of heat balance and thermal efficiency of hot blast stove in No. 2 blast furnace of Jiuquan Iron and Steel [J]. Metallurgy, 2014, 36(04): 22-25. [2017-08-02] DOI: 10.16042/j.cnki.cn621053 / tf.2014.04.025

[7] Gao Pan. (2013): Mathematical physical simulation of oxygen blast furnace technology [D]. Northeastern University.

[8] Liu Wenwen. (2015): Based on statistical process control of large blast furnace hot metal quality control [D]. Shanghai Jiaotong University.

[9] Yang Kun. (2016): Neural network based on the blast furnace molten iron silicon content modelling [D]. Yanshan University.

[10] Hu Dengfei. (2014): Optimization model of blast furnace coal injection based on neural network prediction model [D]. Inner Mongolia University of Science and Technology.

[11] QI Peng.(2010): Study on Prediction of Silicon Content in Hot Metal of Blast Furnace Based on Partial Least Squares [D]. Inner Mongolia University of Science and Technology.

[12] LI Bao Jian, CHENG Chun Tian. (2014): Monthly discharge forecasting using wavelet neural networks with extreme learning machine [J]. Science China (Science Sciences), (12): 2441-2452.

[13] Peter Schmoele, Michael Peters, Hans Bodo Luengen, Zhu Tingting.(2013): Development and prospect of blast furnace ironmaking [J]. World Steel, (01): 35-41.

[14] Guo Yanling, Hu Junge, Zhou Wentao, Zhao Xiaoyan, Wang Xu, Huang Mingming (2014): Effect of coke quality on blast furnace ironmaking [J]. Shanghai Metal, (03): 51-55.

[15] Mu Dong, Wang Chao, Wang Shengchun, Zhou Shengchuan. (2015): Based on parallel simulated annealing algorithm to solve the time-dependent vehicle routing problem [J]. Computer Integrated Manufacturing System, (06): 1626-1636.

[16] LU Yu-ting, LIN Yu-ya, PENG Qiao-zi, WANG Ying-zhe.I (2015): mproved overview and simulated probing of simulated annealing algorithm [J]. College Mathematics, (06): 96-103.

[17] Yue Zhenfang, Gao Yuelin.(2016): Improved teaching and optimization algorithm for simulated simulated annealing [J]. Journal of Henan Normal University (Natural Science Edition), (01): 149-154.

[18] Wang Jie, LI Hui-hui, Peng Jin-zhu. (2016): An Quasirandom Initial Simulated Annealing Particle Swarm Optimization Algorithm [J]. Journal of Zhengzhou University (Natural Science Edition).03): 75-81.

[19] Yan Lei, Ma Jian, Dong Hui, Gao Meng. (2017): Application of genetic algorithm and simulated annealing algorithm in multi-DNA sequence alignment [J]. Journal of Hainan Institute of Tropical and Oceanic Sciences, (02): 64- 69.

[20] Zhou Ping, Li Ruifeng, Guo Dongwei, Wang Hong, Chai Tianyou.(2016): Multi-output support vector regression modelling of multicomponent hot metal mass index in blast furnace iron making process $[\mathrm{J}]$. Control Theory \& Applications, (06): 727-734.

[21] Cui Gui-mei, Cheng history.(2014): Measurement of blast furnace temperature prediction based on distributed neural network model [J]. Journal of Iron and Steel Research, 2014, (06): 27-30.

[22] Cui Gui-mei, Gao Cui-ling, Hou Jia, Chen Zhi-hui, MA Xiang. (2015): A optimization of blast furnace coal injection based on genetic algorithm for BP neural network [J]. Computer Measurement and Control, (05): 1568-1570 + 1574.

[23] Real estate appraisal system based on GIS and BP neural network [J]. Transactions of Nonferrous Metals Society of China 2011, (S3): 626-630.

[24] Tang YuHua, Zhang BaiDa, Wu JunJie, Hu TianJiang, Zhou Jing, Liu Fu Dong. (2013): Parallel architecture and optimization for discrete-event simulation of spike neural networks [J]. Science China (Technological Sciences), (02): 509-517.

[25] Huang Li. (2008): BP neural network algorithm improvement and application research [D]. Chongqing Normal University. 